\title{
Evolutionary games and matching rules
}

\author{
Martin Kaae Jensen ${ }^{1}$ • Alexandros Rigos $^{2}(D$
}

Accepted: 5 April 2018 / Published online: 11 June 2018

(C) The Author(s) 2018

\begin{abstract}
This study considers evolutionary games with non-uniformly random matching when interaction occurs in groups of $n \geq 2$ individuals using pure strategies from a finite strategy set. In such models, groups with different compositions of individuals generally co-exist and the reproductive success (fitness) of a specific strategy varies with the frequencies of different group types. These frequencies crucially depend on the matching process. For arbitrary matching processes (called matching rules), we study Nash equilibrium and ESS in the associated population game and show that several results that are known to hold for population games under uniform random matching carry through to our setting. In our most novel contribution, we derive results on the efficiency of the Nash equilibria of population games and show that for any (fixed) payoff structure, there always exists some matching rule leading to average fitness maximization. Finally, we provide a series of applications to commonly studied normal-form games.
\end{abstract}

Previous versions of the paper have been circulated under the title "Evolutionary Games with Group Selection." We would like to thank Heinrich Nax, Chris Wallace, Jonathan Newton, Matthijs van Veelen, Jörgen Weibull, an anonymous associate editor, two anonymous referees, and participants at the 2012 UECE Lisbon Meeting, the 2014 "Norms, Actions, Games" Conference in London, the 2014 "Controversies in Game Theory" workshop in Zurich and the 2015 Royal Economic Society PhD Meetings and Job Market. All remaining errors are our own.

Martin Kaae Jensen

m.k.jensen@surrey.ac.uk

Alexandros Rigos

http://www.alexrigos.com

1 School of Economics, University of Surrey, Guildford, UK

2 Department of Economics, School of Economics and Management, Lund University, Lund, Sweden 
Keywords Evolutionary game theory · Evolutionarily stable strategy · ESS . Non-uniformly random matching $\cdot$ Assortative matching $\cdot$ Replicator dynamic

\section{Introduction}

The canonical evolutionary game theory model of Maynard Smith and Price (1973) plays an important role in biology, economics, political science, and other fields. Its equilibrium concept, an evolutionarily stable strategy (ESS) describes evolutionary outcomes in environments where populations are large and matching is uniformly random. ${ }^{1}$ Since an ESS is a refinement of Nash equilibrium, it obviously cannot explain any behavioral departure from purely self-serving behavior in the one-shot Nash sense. In particular it cannot account for cooperative behavior in say, a prisoners' dilemma, or shed light on altruism more generally, nor can it account for any other non-Nash behaviors such as spite (Hamilton 1970; Alger and Weibull 2012) or costly punishment (Fehr and Gächter 2000).

In order to explain such deviations from Nash behavior, evolutionary game theory turned to models with a finite number of agents hence departing from the first of the mentioned conditions of Maynard Smith and Price (1973). Thus in Schaffer (1988), the finite set of individuals have "market power" and can influence average fitness while making simultaneous decisions (playing the field). In the model preferred by Maynard Smith (1982) —namely repeated games — a few agents, usually just two, can perfectly monitor and record each others' past actions and condition their strategies hereupon (in evolutionary theory, the repeated games approach is usually referred to as direct reciprocity). Both of these frameworks have led to a large body of research in economics and game theory (see e.g. Alós-Ferrer and Ania 2005; Leininger 2006; Samuelson 2002; Vega-Redondo 1997, and references therein).

Others, beginning with Wright $(1921,1922)$ and his $F$-statistic, focused on studying populations where individuals do not get matched in a uniformly random manner. When matching is non-uniformly random the fitness of an individual will depend on the group of individuals he is matched with, and groups with different compositions will on average meet with varying reproductive success (Kerr and Godfrey-Smith 2002); see also Bergström (2002). Take the prisoners' dilemma. If cooperators have a higher chance compared to defectors to be matched with cooperators, matching is non-uniformly random, and specifically it is in this case assortative. If the matching is assortative enough, cooperators will end up receiving a higher average fitness than defectors and thus positive levels of cooperation can become evolutionarily stable. Assortative matching has also been shown to lead to more cooperative outcomes in Moran processes (Cooney et al. 2016). ${ }^{2}$

Non-uniformly random matching is a realistic description in situations where a large group of individuals cannot perfectly monitor each others' past behaviors but receive

\footnotetext{
1 Intuitively, uniformly random matching means that an individuals' type has no influence on what type of individual he is likely to be matched to.

2 For an overview see Rousset (2004). For a survey of more recent advances in the social sciences, see Newton (2018, Sect. 3).
} 
some signals about opponents' types and exert some influence on the matching process (Wilson and Dugatkin 1997; Bergström 2003). It can also result due to prolonged interaction of individuals in separated groups (Maynard Smith 1964), if individuals are matched according to a "meritocratic matching" process in the sense of Nax et al. (2014), if matching depends on the geographical location of individuals (Eshel et al. 1998; Nowak and May 1992; Skyrms 2004), or if (genetically) similar individuals match assortatively as in models of kin selection (Hamilton 1964; Grafen 1979; Hines and Maynard Smith 1979; Alger and Weibull 2010; Ohtsuki 2010). Several other processes are listed in Bergström (2013) who also shows that the index of assortativity of Bergström (2003) and Wright's $F$-statistic are formally equivalent. In general, the above conditions lead to what biologists refer to as structured populations. ${ }^{3}$

Now, the existing literature on non-uniformly random matching usually deals with special cases - the typical being the two-player, two-strategy case where matching is assortative. Exceptions to this include Kerr and Godfrey-Smith (2002) who study many-player games with two strategies, van Veelen (2011) who uses a setting similar to ours and discusses inclusive fitness, and Alger and Weibull (2016) who develop a general model to investigate the evolutionary stability of preferences. Here we consider the general case and define Nash equilibrium and ESS within the resulting population game (Sandholm 2010, pp. 22-23). The fitness function of the population game is derived from two primitives: a (symmetric, normal-form game) payoff matrix and a function that assigns particular population compositions to group compositions (called a matching rule). Given this structure of fitness functions, we then show how several results known from population games carry through to our setting. In particular, any Nash equilibrium is a steady state for the replicator dynamic, any (Lyapunov) stable steady state for the replicator dynamic is a Nash equilibrium, and any ESS is an asymptotically stable state of the replicator dynamic.

More substantially, we push the literature forward by deriving results on the efficiency of the Nash equilibria of population games. A key point-well known from the prisoners' dilemma-is that under uniformly random matching, Nash equilibrium may be inefficient in the sense that the average fitness of the population is not maximized. Since ESS and Nash equilibrium coincide in evolutionary models based on uniformly random matching, it follows that uniformly random matching generally fails to produce outcomes that are efficient. When matching is non-uniformly random, this raises the following question: If we keep the payoff structure fixed and vary the matching rule, will some matching rule lead to efficiency? Our main result in this regard (Proposition 3) tells us that any efficient outcome will in fact be a Nash equilibrium under some matching rule. Such efficient outcomes could, for example, be reached endogenously by populations who can influence the matching process. ${ }^{4}$

\footnotetext{
3 An interesting study is that of van Veelen et al. (2012) who use a model where interactions are repeated and the population is also structured. They find that an assortative population structure significantly increases cooperation levels.

${ }^{4}$ Nax and Rigos (2016) show that while this is true for certain classes of games, it is not always the case. In a similar setting, $\mathrm{Wu}$ (2016) studies coordination games in a stochastic setting and shows that the Pareto dominant outcome is always stochastically stable. Studying the evolution of (other-regarding) preferences, Newton (2017) shows that if assortativity itself is subject to evolution, Pareto inefficient behavior can be evolutionarily stable.
} 
The structure of the paper is as follows. Section 2 describes the general setup, introduces matching rules, and defines Nash equilibrium and ESS. Section 3 contains our main theoretical results. Section 4 provides a number of applications in two-player, two-strategy normal-form games. Finally, Sect. 5 concludes.

\section{Population games under matching rules}

In this section we formulate the basic model as a population game under the replicator dynamic (see Taylor and Jonker 1978, Sandholm 2010, pp. 22-23). At each point in time there is a unit mass of individuals each of whom follows one of $m \in \mathbb{N}$ pure strategies. The individuals are drawn into groups of size $n \in \mathbb{N}$ according to a particular protocol which we term a matching rule and describe in detail below. ${ }^{5}$ In the groups, individuals execute their strategies and receive payoffs (fitness) determined by the composition of strategies in the groups they are drawn into. The average fitness level of individuals following a given pure strategy across the groups determines the strategy's (overall) fitness and therefore the proportion of individuals that follow that pure strategy at the next point in time. This leads to a dynamical system of the replicator variety which we study in continuous time.

\subsection{Groups and matching rules}

Let $M=\{1, \ldots, m\}$ denote the set of pure strategies. An individual who follows (pure) strategy $i \in M$ is interchangeably said to be of type $i$ or an $i$-type. As mentioned, $n$ is the (finite) group size. A group type $g \in G \equiv\left\{\hat{g} \in \mathbb{N}^{m}: \sum_{i \in M} \hat{g}_{i}=n\right\}$ is a vector that specifies a number of individuals $g_{i}$ of each type $i=1, \ldots, m$. With group size $n$ and $m$ pure strategies, there are $\frac{(n+m-1) !}{n !(m-1) !}$ distinct group types (see Aigner 2007, p. $15)$. Hence the cardinality of the set of group types $G$ is $\gamma \equiv \frac{(n+m-1) !}{n !(m-1) !}$. To simplify notation in the following define $P_{i g}=\frac{g_{i}}{n} \in[0,1]$, which is the fraction of individuals in group type $g$ that are of type $i$. The matrix with typical element $P_{i g}$ is denoted by $P \in \mathbb{R}^{m \times \gamma}$.

The frequency distribution of different (individual) types at a moment in time is called a population state and denoted by $x \in \Delta_{m}$ (throughout $\Delta_{m} \equiv\left\{x \in \mathbb{R}_{+}^{m}\right.$ : $\left.\left.\sum_{i=1}^{m} x_{i}=1\right\}\right)$. Note that the $i$ 'th element in a population state $x$ simply is the proportion of $i$-type individuals in the population. The frequency distribution of group types is called a group state and denoted $z \in \Delta_{\gamma}$ with $z_{g}$ the proportion of groups of type $g$. A matching rule is a function that maps population states into group states.

Definition 1 (Matching Rule) A matching rule is a function $f: \Delta_{m} \rightarrow \Delta_{\gamma}$ such that

$$
x_{i}=\sum_{g \in G} P_{i g} f_{g}(x) \quad \text { for all } i \in M
$$

\footnotetext{
5 Our notion of a matching rule generalizes what Kerr and Godfrey-Smith (2002, p. 484) call a "rule of group assembly" to more than 2 pure strategies (the concept is also related to Bergström 2003, as returned to below).
} 
or, more concisely,

$$
x=\operatorname{Pf}(x) .
$$

Note that $P_{i g} f_{g}(x)$ is the fraction of the (total) population that is of type $i$ and is allocated into a $g$-type group under the matching rule $f$. So (1) (or equivalently, (2)), ensures that the fraction of $i$-type individuals allocated into the different groups equals the fraction $x_{i}$ of $i$-type individuals that are actually present in the population. This of course is an entirely natural consistency requirement given our interpretation of matching rules as mappings that allocate individuals into groups.

Let $w_{i g}$ denote the fraction of $i$-type individuals that is allocated to a $g$-type group. It is easily seen that,

$$
w_{i g}(x)=\frac{P_{i g} f_{g}(x)}{x_{i}},
$$

where of course $x_{i}>0$ must hold (otherwise there would be no individuals of type $i$ in the first place). Note that $w_{i g}(x)$ (also) may be interpreted as the probability that an individual of type $i$ is drawn into a group of type $g$.

\subsection{Payoffs and equilibrium}

Having described how agents are allocated into groups by means of a matching rule $f$, we now formulate the interaction as a population game (see Sandholm 2010, pp. 22-23 for example). Recall from the beginning of Sect. 2.1, that a group type $g$ is a vector that specifies how many individuals of each of the $m$ types reside in the group. Within each group individuals receive payoff according to a symmetric normal-form game. In such games the payoff of each player depends only on the strategy he follows and the number of other players that follow each of the $m$ strategies (as opposed to who uses each strategy). The game is represented by a matrix $A \in \mathbb{R}^{n \times \gamma}$. Its typical entry $A_{i g} \in \mathbb{R}$ is interpreted as the payoff a type $i$ individual receives upon executing his strategy in group type $g .6$

Since $A_{i g}$ is the payoff to an individual of type $i$ in group type $g$ and $w_{i g}(x)$ is the probability that an $i$-type individual ends up in a group type $g$ (see the paragraph following Definition 1), the (ex-ante) population-wide expected payoff/fitness to an $i$-type individual when the population state is $x$ (and $x_{i}>0$ ) equals

$$
F_{i}(x)=\sum_{g \in G} w_{i g}(x) A_{i g}
$$

This defines fitness functions $F_{i}:$ int $\left(\Delta_{m}\right) \rightarrow \mathbb{R}$, that are the coordinate functions of $F:$ int $\left(\Delta_{m}\right) \rightarrow \mathbb{R}^{m}$.

We extend the definition of $F$ to include the boundary of $\Delta_{m}$ by setting $w_{i g}(x)=$ $\lim _{\tilde{x}_{i} \downarrow 0} P_{i g} f_{g}(\tilde{x}) / \tilde{x}_{i}$ whenever $x \in \operatorname{bd}_{i}\left(\Delta_{m}\right){ }^{7}$ We will assume that the matching rule

\footnotetext{
${ }^{6}$ Of course, some of the entries in this matrix are meaningless-for example the payoff to an individual of type $i$ when he is found in a group in which all members are of type $j \neq i$-but this will create no problems in what follows.

${ }^{7}$ We define $\operatorname{bd}_{i}\left(\Delta_{m}\right) \equiv\left\{x \in \Delta_{m}: x_{i}=0\right\}$.
} 
$f$ is such that $F$ can be extended to a Lipschitz continuous function on $\Delta_{m}$. Now notice that under condition (1), if $P_{i g}>0$ (equivalently $g_{i}>0$ ), then $\lim _{x_{i} \rightarrow 0} f_{g}(x)=0$ and so $\lim _{\tilde{x}_{i} \downarrow 0} f_{g}(\tilde{x}) / \tilde{x}_{i}$ precisely is the $i$ 'th partial (upper) derivative of $f_{g}, \partial_{i}^{+} f_{g}(x)$ when $x_{i}=0$. Hence $w_{i g}(x)=P_{i g} \partial_{i}^{+} f_{g}(x)$ when $x_{i}=0$.

Given the above observations, the following condition is sufficient to ensure Lipschitz continuity of $F: \Delta_{m} \rightarrow \mathbb{R}^{m}$.

Assumption 1 The matching rule $f$ satisfies the following:

(i) $f$ is Lipschitz continuous

(ii) if $x \in \operatorname{bd}_{i}\left(\Delta_{m}\right)$, then $\partial_{i}^{+} f_{g}(x)$ exist for all $(i, g) \in M \times G$ such that $g_{i}>0$.

Note that the differentiability requirement is satisfied trivially if the matching rule is differentiable at the boundary of $\Delta_{m}$.

In this way, through condition (4), a payoff matrix $A$ and a matching rule $f$ define the payoff function $F$. We identify the population game induced by $A$ and $f$ by this payoff function and write $F^{A, f}$ to refer explicitly to the payoff matrix and matching rule. A Nash equilibrium of the induced game is defined as usual (Sandholm 2010, p. 24).

Definition 2 (Nash equilibrium) A Nash equilibrium of the induced population game $F^{A, f}$ is a population state $x^{*} \in \Delta_{m}$ such that for $i \in M$ with $x_{i}^{*}>0$ :

$$
F_{i}^{A, f}\left(x^{*}\right) \geq F_{k}^{A, f}\left(x^{*}\right) \text { for all } k \in M
$$

Following standard arguments, continuity of $F^{A, f}$ implies that a Nash equilibrium exists. Therefore, a population game under a matching rule $f$ that satisfies Assumption 1 is guaranteed to have a Nash equilibrium.

In the evolutionary game theory literature, the key equilibrium refinement concept is that of an Evolutionarily Stable Strategy or State (ESS). ESS is usually defined in games with uniformly random matching and in the special case when $n=2$ (see for example Hofbauer and Sigmund 1998, p. 63). Since matching can be non-uniformly random, there are possible nonlinearities introduced into the payoff function through the matching rule $f$. Thus, the appropriate definition of an ESS needs to have the local character of Pohley and Thomas (1983). We formulate the definition by means of a uniform invasion barrier (see e.g. Sandholm 2010, p. 276) as in this manner what "local" means is made clear.

Definition 3 (ESS) An ESS of the induced population game $F^{A, f}$ is a population state $\hat{x} \in \Delta_{m}$ for which there exists $\bar{\varepsilon}>0$ such that for all $y \in \Delta_{m} \backslash\{\hat{x}\}$ and all $\varepsilon \in(0, \bar{\varepsilon})$

$$
\hat{x} \cdot F^{A, f}(\varepsilon y+(1-\varepsilon) \hat{x})>y \cdot F^{A, f}(\varepsilon y+(1-\varepsilon) \hat{x}) .
$$

Assumption 1 ensures that $F^{A, f}$ is continuous. Therefore, if it holds the standard result that an ESS is a refinement of Nash equilibrium applies.

Proposition 1 Let $F^{A, f}$ be the population game induced by payoff matrix $A$ and matching rule $f$. Let also $f$ satisfy Assumption 1 . Then any ESS of $F^{A, f}$ is a Nash equilibrium. 
Proof By way of contradiction, assume that some $\hat{x} \in \Delta_{m}$ is an ESS but not a Nash equilibrium. Then, there exists some $y \in \Delta_{m}$ such that $(y-\hat{x}) \cdot F^{A, f}(\hat{x})>$ 0 . But from the definition of an ESS, there exists some $\bar{\varepsilon} \in(0,1)$ such that $(y-$ $\hat{x}) \cdot F^{A, f}(\varepsilon y+(1-\varepsilon) \hat{x})<0$ for all $\varepsilon \in(0, \bar{\varepsilon})$. As explained before, the two conditions of Assumption 1, imply continuity of $F^{A, f}$. By continuity therefore $(y-$ $\hat{x}) \cdot F^{A, f}(\hat{x}) \leq 0$, a contradiction.

\subsection{Examples of matching rules}

Before turning to describe the dynamical system that will determine the evolution of the population in our model, we provide some examples of matching rules. Note that all of the examples satisfy Assumption 1.

\subsubsection{Complete segregation}

Different strategies do not mix. All individuals are allocated into groups with only individuals of the same type and thus all groups contain a single type of individuals each ( $n$ individuals that follow the same strategy). The group types that have $n$ individuals of the same type get a non-negative frequency whereas all other kinds of groups get a frequency of zero. Due to the consistency requirements for matching rules, we get that the group type that contains $n i$-types should get a frequency of $x_{i}$. So, formally, the matching rule for complete segregation is the following.

$$
\begin{aligned}
& f_{g}(x)=x_{i}, \quad \text { if } P_{i g}=1 \\
& f_{g}(x)=0 \text {, otherwise. }
\end{aligned}
$$

For example, say Say $n=2$ and $m=2$ with the two strategies being $C$ and $D$. There are three group types: $\{C C\},\{C D\}$ and $\{D D\}$. The matching rule for complete segregation takes the form

$$
f_{\{C C\}}\left(x_{C}, x_{D}\right)=x_{C} \quad f_{\{C D\}}\left(x_{C}, x_{D}\right)=0 \quad f_{\{D D\}}\left(x_{C}, x_{D}\right)=x_{D} .
$$

\subsubsection{Uniformly random matching}

Let us define an opponent profile to be a collection $v=\left(v_{j} \in \mathbb{N}\right)_{j \in M}$ such that $\sum_{j=1}^{m} v_{j}=n-1$. We denote the set of all opponent profiles by $\mathbb{O}$. The set $\mathbb{O}$ consists of all possible combinations of types of other individuals that an individual can find in the group in which he is matched.

A matching rule $f$ is going to be called uniformly random if the ex-ante probability of an individual (conditional on her type) to face an opponent profile $v$ is independent of the individual's type, for all $v \in \mathbb{O}$. If this is the case, then the frequencies of group types will follow a multinomial distribution (see for example Lefebvre 2007, p. 22):

$$
f_{g}(x)=\frac{n !}{\prod_{j=1}^{m} g_{j} !} \prod_{j=1}^{m} x_{j}^{g_{j}}
$$


One can easily verify that expression (8) indeed describes a matching rule i.e. that it satisfies (1). To show that the property described above holds for the matching rule of equation (8), first observe that an opponent profile $v \in \mathbb{O}$ along with an individual-type $i \in M$ uniquely define a group-type. Since $g_{j}$ is the number of $j$-type individuals in a $g$-type group, the group-type created by adding an $i$-type individual to an opponent profile $v$ will have the following structure: ${ }^{8}$

$$
g_{j}=\left\{\begin{array}{ll}
v_{j}+1 & \text { if } j=i \\
v_{j} & \text { if } j \in M \backslash\{i\}
\end{array} .\right.
$$

So, the probability of an $i$-type individual to end up in a group where she faces the opponent profile $v$ is the same as the probability of an $i$-type individual to end up in a group with a structure given by (9).

Now recall that the probability of an $i$-type individual (conditional on her type) to end up in a $g$-type group is given by $w_{i g}(x)$ of equation (3). Using this formula for the matching rule of equation (8) yields

$w_{i g}(x)=\frac{P_{i g}}{x_{i}} f_{g}(x)=\frac{g_{i}}{n x_{i}} \frac{n !}{\prod_{j=1}^{m} g_{j} !} \prod_{j=1}^{m} x_{j}^{g_{j}}=\frac{x_{i}^{g_{i}-1}}{\left(g_{i}-1\right) !} \frac{(n-1) !}{\prod_{j \in M \backslash\{i\}} g_{j} !} \prod_{j \in M \backslash\{i\}} x_{j}^{g_{j}}$.

Applying this for the particular group that has the composition given by (9) and noting that $v_{i}=g_{i}-1$ and $v_{j}=g_{j}$ for all $j \neq i$, we get that the probability of an $i$-type to face an opponent profile $v$ is

$$
\frac{x_{i}^{v_{i}}}{v_{i} !} \frac{(n-1) !}{\prod_{j \in M \backslash\{i\}} v_{j} !} \prod_{j \in M \backslash\{i\}} x_{j}^{v_{j}}=\frac{(n-1) !}{\prod_{j=1}^{m} v_{j} !} \prod_{j=1}^{m} x_{j}^{v_{j}}
$$

which only depends on the opponent profile $v$ and the population state $x$ i.e. it is independent of the indiviudal's type $i$. Therefore expression (8) describes a uniformly random matching rule.

Notice that for $m=2$, the uniformly random matching rule becomes

$$
f_{g}\left(x_{1}, x_{2}\right)=\frac{n !}{g_{1} ! g_{2} !} x_{1}^{g_{1}} x_{2}^{g_{2}} \text {. }
$$

That is it boils down to the binomial distribution (see Kerr and Godfrey-Smith 2002, p. 484). And for $n=2$, we get

$$
f_{(2,0)}\left(x_{1}, x_{2}\right)=x_{1}^{2} \quad f_{(1,1)}\left(x_{1}, x_{2}\right)=2 x_{1} x_{2} \quad f_{(0,2)}\left(x_{1}, x_{2}\right)=x_{2}^{2} .
$$

\footnotetext{
8 Note that the group-types that are formed by two different individual-types facing the same opponent profile will be different.
} 


\subsubsection{Constant index of assortativity (2 strategies)}

Bergström (2003) studies 2-person prisoners' dilemma population games by using the "index of assortativity" which he defines as "the difference between the probability that a C-strategist meets a C-strategist and the probability that a D-strategist meets a C-strategist." In terms of notation used in this paper this means that the index of assortativity when the population state is $\left(x_{C}, x_{D}\right)$ will be:

$\alpha\left(x_{C}, x_{D}\right)=w_{C\{C C\}}\left(x_{C}, x_{D}\right)-w_{D\{C D\}}\left(x_{C}, x_{D}\right)=\frac{f_{\{C C\}}\left(x_{C}, x_{D}\right)}{x_{C}}-\frac{f_{\{C D\}}\left(x_{C}, x_{D}\right)}{2 x_{D}}$.

Bergström goes on to analyze prisoners' dilemma games under assortative matching rules for which the index of assortativity $\alpha$ is constant for all values of $x$. As one easily verifies, the matching rule corresponding to a constant index of assortativity $\alpha$ is:

$$
\begin{aligned}
& f_{\{C C\}}\left(x_{C}, x_{D}\right)=\alpha x_{C}+(1-\alpha) x_{C}^{2} \\
& f_{\{C D\}}\left(x_{C}, x_{D}\right)=2(1-\alpha) x_{C} x_{D} \\
& f_{\{D D\}}\left(x_{C}, x_{D}\right)=\alpha x_{D}+(1-\alpha) x_{D}^{2} .
\end{aligned}
$$

In the case of $\alpha=0$ the rule coincides with the random matching rule and in the case of $\alpha=1$ it coincides with the complete segregation rule (for both of these statements we need $n=m=2$, i.e., two players and two strategies).

\subsection{4 (Almost) constant index of dissociation}

It is not possible to use the previous definition of a constant index of assortativity rule for negative values of $\alpha$-i.e. to dissociative matching-without violating the consistency condition (1) that defines matching rules. Indeed, if such a "constant index of dissociation" rule is imposed without any changes, the matching rule would necessarily violate (1) when $x_{C}$ is close to 0 or to 1 : in the former case there are not enough $C$-types with whom the $D$-types should be matched and vice versa when $x_{C}$ is close to $1 .^{9}$ So, to consider a matching rule with an index of dissociation that is constant whenever it is possible, one must "tweak" the construction slightly near the boundary. In the matching rule we propose, we deal with that by matching as many individuals as possible in mixed groups and the remaining individuals in homogeneous groups. So, a matching rule with an (almost) constant index of dissociation $\beta \in[0,1]$ is given by the following:

$$
\begin{aligned}
f\left(x_{C}, x_{D}\right)= & \left(f_{\{C C\}}\left(x_{C}, x_{D}\right), f_{\{C D\}}\left(x_{C}, x_{D}\right), f_{\{D D\}}\left(x_{C}, x_{D}\right)\right) \\
& = \begin{cases}\left(0,2 x_{C}, 1-2 x_{C}\right), & x_{C} \in\left[0, \frac{\beta}{1+\beta}\right] \\
\left((1+\beta) x_{C}^{2}-\beta x_{C}, 2(1+\beta) x_{C} x_{D},(1+\beta) x_{D}^{2}-\beta x_{D}\right), & x_{C} \in\left(\frac{\beta}{1+\beta}, \frac{1}{1+\beta}\right) . \\
\left(1-2 x_{D}, 2 x_{D}, 0\right), & x_{C} \in\left[\frac{1}{1+\beta}, 1\right]\end{cases}
\end{aligned}
$$

\footnotetext{
${ }^{9}$ More specifically, this happens for $x_{C} \in\left(0, \frac{-\alpha}{1-\alpha}\right) \cup\left(\frac{1}{1-\alpha}, 1\right)$ when $\alpha \in[-1,0)$.
} 


\subsubsection{Constant index of assortativity ( $m$ strategies)}

The constant index of assortativity rule of 2.3.3 can be extended to 2-player games with more than two strategies (Bergström 2013) where the index $\alpha$ denotes the proportion of individuals of any given type to enter a pool that consists only of individuals of the same type (and get matched to individuals from within their pool). The remaining proportion $(1-\alpha)$ enters a common pool where individuals match uniformly randomly. This description generates the following matching rule in our formulation:

$$
\begin{aligned}
& f_{\{i i\}}(x)=\alpha x_{i}+(1-\alpha) x_{i}^{2} \\
& f_{\{i j\}}(x)=2(1-\alpha) x_{i} x_{j} \quad \text { if } j \neq i .
\end{aligned}
$$

\subsubsection{Constant index of uniform group formation ( $n$ players, 2 strategies)}

Following a similar way of thinking as in Sect. 2.3.5, an assortative matching rule can be extended to $n$-player games with two strategies/types. The rule of Eq. (10) describes the following process: a proportion $\alpha$ of each of the two types enters a pool that consists only of individuals of the same type and (uniform) $n$-sized groups are formed from within these two pools. The remaining proportion $(1-\alpha)$ enters a common pool where individuals are drawn to form $n$-sized groups uniformly randomly. This leads to the matching rule being

$$
\begin{aligned}
& f_{(0, n)}\left(x_{C}, x_{D}\right)=\alpha x_{D}+(1-\alpha) x_{D}^{n} \\
& f_{(k, n-k)}\left(x_{C}, x_{D}\right)=(1-\alpha) \frac{n !}{k !(n-k) !} x_{C}^{k} x_{D}^{n-k} \text { for } k=1,2, \ldots, n-1 \\
& f_{(n, 0)}\left(x_{C}, x_{D}\right)=\alpha x_{C}+(1-\alpha) x_{C}^{n} .
\end{aligned}
$$

\subsection{Dynamics}

Let $x^{t} \in \Delta_{m}$ denote the population state at time $t$. At time $t$, the population is allocated into groups according to the matching rule $f$, hence $f\left(x^{t}\right) \in \Delta_{\gamma}$ is the resulting frequency distribution of group types. Regardless of which group an individual of type $i$ ends up in, he will mechanically follow the strategy of his type (as inherited from the parent) and fitness will be distributed accordingly. The average fitness that an $i$-type individual receives is given by (4), repeated here for the reader's convenience and with explicit reference to the payoff matrix and matching rule:

$$
F_{i}^{A, f}\left(x^{t}\right)=\sum_{g \in G} w_{i g}(x) A_{i g} .
$$

The average fitness across the entire population is,

$$
\bar{F}^{A, f}\left(x^{t}\right)=\sum_{i=1}^{m} x_{i}^{t} F_{i}^{A, f}\left(x^{t}\right)=x^{t} \cdot F^{A, f}\left(x^{t}\right) .
$$


All that now remains is to describe how these fitnesses determine the next generation. For this, we use the continuous-time replicator dynamic (Taylor and Jonker 1978) which formalizes the idea that the rate at which the proportion of $i$-type individuals grows is equal to the amount by which type- $i$ average fitness $\left(F_{i}^{A, f}\right)$ exceeds the population-wide average fitness $\left(\bar{F}^{A, f}\right)$.

Definition 4 The continuous-time replicator dynamic of the induced population game $F^{A, f}$ is given by the equations:

$$
\dot{x}_{i}^{t}=x_{i}^{t}\left(F_{i}^{A, f}\left(x^{t}\right)-\bar{F}^{A, f}\left(x^{t}\right)\right) \quad \text { for } i \in M
$$

with $F_{i}^{A, f}$ and $\bar{F}^{A, f}$ defined in equations (11) and (12), respectively.

Definition 5 A steady state of the induced population game $F^{A, f}$ is a rest point of the dynamical system (13).

Notice that as we do not assume any linearity of the matching rule $f$, the fitness functions $F_{i}^{A, f}, i=1, \ldots, m$ will typically be nonlinear. This is in contrast with the linear fitness functions obtained under uniformly random matching. Different notions of stability such as Lyapunov and asymptotic stability are defined as usual in either case, and the associated steady states (if any) are said to be Lyapunov stable, asymptotically stable, and so on. Since any uniform population state-i.e., any state where all individuals are of the same type-will be a steady state, it is clear that stability must be considered or else the model will have no predictive power.

\section{Results}

In this section we provide our main theoretical results. First we establish that several well-known results from the population games literature extend to games induced by matching rules as long as the latter are well-behaved. Secondly, we show that efficient outcomes can always be supported as Nash equilibria of population games induced by appropriately selected matching rules.

\subsection{Dynamic stability and equilibrium}

In evolutionary models with uniformly random matching, there is a close relationship between dynamic models of the replicator type and game theoretic concepts such as Nash equilibrium and evolutionarily stable strategies (e.g. Hofbauer and Sigmund 1998, Theorem 7.2.1; Weibull 1995, Proposition 3.10). ${ }^{10}$ Given our formalization of matching rules, similar results hold for population games induced by well-behaved (satisfying Assumption 1) non-uniformly random matching rules. In particular (i) any Nash equilibrium is a steady state of the replicator dynamic, (ii) any Lyapunov stable

\footnotetext{
${ }^{10}$ For similar results under even broader classes of dynamics, see Ritzberger and Weibull (1995).
} 
state as well as any any limit of an interior orbit under the replicator dynamic is a Nash equilibrium, and (iii) any evolutionarily stable strategy of $F^{A, f}$ is asymptotically stable for the associated replicator dynamic. ${ }^{11}$

The next proposition shows that population games induced by the uniformly random matching rule of Sect. 2.3.2 have the same steady states and ESS as their standard normal-form game counterparts. In this way our concept of matching rules extends the scope of the tools of evolutionary game theory in a consistent manner.

Proposition 2 Let $F^{A, f}$ be the population game induced by payoff matrix $A$ and the uniformly random matching rule $f$, given by Eq. (8). Then, the set of Nash equilibria of $F^{A, f}$ coincides with the set of symmetric Nash equilibria of the underlying normal form game A. Moreover, the set of ESS of $F^{A, f}$ coincides with the set of ESS of A.

Proof See Appendix B.1.

\subsection{Matching rules and efficiency}

Assortative matching has been shown to be able to explain behavioral traits such as altruism or cooperation which cannot arise in Nash equilibrium and so cannot be favored by natural selection if matching is uniformly random, as seen in Proposition 2 (e.g. Alger and Weibull 2013, 2016). Importantly, such departures from self-regarding behavior may be more efficient than the outcomes under uniformly random matching in the sense that the average fitness may be higher. The classical example here is the prisoners' dilemma where the outcome of uniformly random matching yields lower average fitness than outcomes under assortative matching (see Sect. 4 and also Bergström 2002). In what follows, the welfare notion that we have in mind is a utilitarian one. Thus, efficiency will be measured by the level of average fitness in the population.

The observation that uniformly random matching-or for that matter any other specifically given matching rule $f$-may not maximize average fitness in a Nash equilibrium $x^{*}$ also remains valid if instead of Nash equilibria we focus on ESS. Thus, evolution under non-uniformly random matching certainly does not imply average fitness maximization. The interesting next question therefore is whether for a fixed underlying normal form game $A$ there exists some matching rule under which average fitness will be maximized at a Nash equilbrium of $F^{A, f}$. When discussing this topic it is important to understand that when $f$ is varied, not only does the set of Nash equilibria (and ESS and also, the set of steady states of the replicator dynamics) change- the efficiency level $\bar{F}^{A, f}(x)$ will also change at any given population state $x$. So if some population state maximizes average fitness but is not a Nash equilibrium under some matching rule $f^{\prime}$, it could be a Nash equilibrium under a different matching rule $f^{\prime \prime}$ but no longer maximize average fitness. Any sensible discussion must therefore consider the joint selection of a population state and matching rule as captured by the following definition.

11 The matching rule $f$ satisfying Assumption 1 guarantees Lipschitz continuity of the fitness functions and makes the dynamic (13) imitative. Therefore, one can apply Proposition 5.2.1 of Sandholm (2010, p. 146) to show (i), Theorem 8.1.1 (ibid., 272) to show (ii), and Theorem 8.4.1 (ibid., 283) to show (iii). 
Definition 6 (Evolutionary Optimum) Let $A$ be a symmetric $n$-player, $m$-strategy normal form game. A population state $x^{*} \in \Delta_{m}$ together with a matching rule $f^{*}$ is said to be an evolutionary optimum if

$\bar{F}^{A, f^{*}}\left(x^{*}\right) \geq \bar{F}^{A, f}(x)$ for all $(x, f) \in \mathbb{E}=\left\{(x, f): x\right.$ is a steady state of $\left.F^{A, f}\right\}$.

Intuitively, a population state $x^{*}$ and a matching rule $f^{*}$ form an optimum if they lead to maximum average fitness among all population state/matching rule combinations that satisfy the steady state restriction. Note that the restriction to steady states is entirely natural here: any population state that is not a steady state of the replicator dynamics would immediately be "destroyed" by natural selection. ${ }^{12}$ Given these definitions, we can now answer the previous question:

Proposition 3 Let $\left(x^{*}, f^{*}\right)$ be an evolutionary optimum. Then there exists a matching rule $h$ which satisfies Assumption 1, such that $x^{*}$ is a Nash equilibrium under $h$ and $\left(x^{*}, h\right)$ is an evolutionary optimum. In particular, $\bar{F}^{A, h}\left(x^{*}\right)=\bar{F}^{A, f^{*}}\left(x^{*}\right)$.

\section{Proof See Appendix B.2.}

We obtain the result of Proposition 3 by constructing the matching rule $h$ so that types that are not in the support of $x^{*}$ are matched in homogeneous groups, away from other types. In this way these types cannot be receiving higher fitness than the average fitness at $x^{*}$ as $\left(x^{*}, f^{*}\right)$ is an evolutionary optimum.

Proposition 3 is telling us that any evolutionary optimum can be attained in the evolutionary environment through some matching rule. That this should be so is easy to see in simple cases. In most standard games (including some of those considered in this paper), there is a premium on coordination/uniformity, and so what is needed in order to reach an evolutionary optimum is a sufficiently high level of assortativity. In games where there is a premium on agents in a group being different-e.g., due to specialization - it will instead be a sufficiently high degree of dissociation that leads to evolutionary optimality. It is not obvious that proposition 3 should hold in the latter case, to say nothing of cases where neither assortative nor dissociative matching rules do the trick.

\section{Applications}

In this section we apply the concepts that were developed earlier in the paper to analyze some 2-player 2-strategy symmetric, normal form games that are frequently encountered in the literature under matching rules that have a constant index of assortativity (see Sect. 2.3.3). Namely, we consider the Hawk-Dove (HD) game, the Stag Hunt (SH), and the Prisoners' Dilemma (PD). In all games considered, there is a "cooperative" strategy in the sense that use of this strategy by one player increases the payoff of the player's opponent. Table 1 provides the names by which the two strategies are referred

\footnotetext{
12 Note in this connection that any uniform population state is a steady state (in fact, any uniform population state is a steady state under any matching rule).
} 
Table 1 List of strategy names and defining conditions for the $2 \times 2$ games considered

\begin{tabular}{llll}
\hline Game & Cooperative strategy $(C)$ & Other strategy $(D)$ & Defining conditions \\
\hline HD & Dove & Hawk & $A_{D 2}>A_{C 1}>A_{C 2}>A_{D 3}$ \\
SH & Stag & Hare & $A_{C 1}>A_{D 2} \geq A_{D 3}>A_{C 2}$ \\
PD & Cooperate & Defect & $A_{D 2}+A_{D 3}>A_{C 1}+A_{C 2}$ \\
\hline
\end{tabular}

Table 2 Defining conditions for the three PD game categories (along with $A_{D 2}>A_{C 1}>$ $\left.A_{D 3}>A_{C 2}\right)$

\begin{tabular}{ll}
\hline Sub-additive & $A_{C 1}+A_{D 3}<A_{D 2}+A_{C 2}$ \\
Linear & $A_{C 1}+A_{D 3}=A_{D 2}+A_{C 2}$ \\
Super-additive & $A_{C 1}+A_{D 3}>A_{D 2}+A_{C 2}$ \\
\hline
\end{tabular}

to in the literature for each of the games along with the games' defining conditions. ${ }^{13}$ To simplify notation, we will always refer to the Cooperative strategy as $C$ and to the other strategy as $D$. More than that, we use the following numbers to index the three possible group types: group-type 1 consists of two $C$-type individuals, group-type 2 is the mixed group-type and group-type 3 consists of two $D$-type individuals. Finally, when calculating equilibria and steady states for the population games, we will use $x$ to denote the proportion of the population that follows strategy $C$. Example payoff matrices of the game classes that are considered here are found in Table 3 . In the Appendix we develop a method to find Nash equilibria and ESS in $2 \times 2$ population games induced by matching rules and to depict average fitness contours.

Interestingly, in the case of a constant index of assortativity rule, the payoffs from the two strategies are such that it is as if the individuals in the population are playing a $2 \times 2$ game with altered payoffs under uniformly random matching. This is shown in Table 4. The reason for which this is possible with the constant index of assortativity matching rule is because $f_{2}$ is proportional to $x(1-x)$ (see Sect. 2.3.3) and thus the appropriate terms in the payoff functions $F_{i}^{A, f}$ conveniently cancel out. Such a simple transformation is not possible with more complicated matching rules.

Using the transformed game of Table 4, we can characterize the Nash equilibria and ESS of the population game using the definitions of the concepts: when $\alpha<1$ an $x \in(0,1)$ is a Nash equilibrium iff

$$
x\left(A_{C 2}-A_{C 1}+A_{D 2}-A_{D 3}\right)=A_{C 2}-A_{C 1}+\frac{A_{C 1}-A_{D 3}}{1-\alpha},
$$

and when $\alpha=1$ any $x \in(0,1)$ is a Nash equilibrium iff $A_{C 1}=A_{D 3}$. An $x \in$ $(0,1)$ is an ESS if - together with the above condition - the following hold: $\alpha\left(A_{D 2}-\right.$

\footnotetext{
13 Following Bergström (2003) we identify three cases of the PD game: those who correspond to public goods games with (i) sub-additive, (ii) linear, and (iii) super-additive production functions. The conditions for the three cases of PD are shown in Table 2.
} 
Table 3 Example payoff matrices for the $2 \times 2$ games considered

\begin{tabular}{lll}
\hline & C & D \\
\hline (a) HD & & \\
C & 10,10 & 8,18 \\
D & 18,8 & 6,6 \\
(b) SH & & \\
C & 10,10 & 0,7 \\
D & 7,0 & 6,6 \\
(c) PD (sub-additive) & & \\
C & 10,10 & 5,18 \\
D & 18,5 & 6,6 \\
(d) PD (linear) & & \\
C & 10,10 & 5,11 \\
D & 11,5 & 6,6 \\
(e) PD (super-additive) & & 3,11 \\
C & 10,10 & 6,6 \\
D & 11,3 & \\
\hline
\end{tabular}

Table 4 The transformation of symmetric $2 \times 2$ normal-form games resulting from a constant index of assortativity rule. The payoffs displayed are for the row player

\begin{tabular}{|c|c|c|c|c|c|c|}
\hline & $\mathrm{C}$ & $\mathrm{D}$ & & & $\mathrm{C}$ & $\mathrm{D}$ \\
\hline $\mathrm{C}$ & $A_{C 1}$ & $A_{C 2}$ & $\longrightarrow$ & $\mathrm{C}$ & $A_{C 1}$ & $\alpha A_{C 1}+(1-\alpha) A_{C 2}$ \\
\hline $\mathrm{D}$ & $A_{D 2}$ & $A_{D 3}$ & & $\mathrm{D}$ & $\alpha A_{D 3}+(1-\alpha) A_{D 2}$ & $A_{D 3}$ \\
\hline
\end{tabular}

$\left.A_{D 3}\right)<A_{D 2}-A_{C 1}$ and $\alpha\left(A_{C 1}-A_{C 2}\right)>A_{D 3}-A_{C 2} \cdot{ }^{14}$ Moreover, $x=0$ is a Nash equilibrium iff $\alpha\left(A_{C 1}-A_{C 2}\right) \leq A_{D 3}-A_{C 2}$, whereas $x=1$ is a Nash equilibrium iff $\alpha\left(A_{D 2}-A_{D 3}\right) \geq A_{D 2}-A_{C 1}$. Each of the two uniform population states is an ESS if the respective inequality holds strictly.

Our analysis shows that the $2 \times 2$ games considered have fundamentally different comparative statics with respect to the assortativity parameter $\alpha$ depending on whether $A_{D 2}+A_{C 2}$ is greater or less than $A_{C 1}+A_{D 3}$. In particular, if $A_{D 2}+A_{C 2}>A_{C 1}+A_{D 3}$ (which is the case in the HD and sub-additive PD), then for any value of $\alpha$ the induced population game $F^{A, f}$ has a unique Nash equilibrium which is also an ESS. On the contrary, for normal-form games with $A_{D 2}+A_{C 2}<A_{C 1}+A_{D 3}$ (which is the case in the SH and super-additive PD) there are regions of the assortativity parameter $\alpha$ for which the replicator dynamic equation is bistable and the induced population game has three Nash equilibria: one ESS at $x=1$ where the whole population follows the cooperative strategy $(C)$, one ESS at $x=0$ where the whole population follows the other strategy $(D)$ and a Nash equilibrium which is not an ESS where part of

\footnotetext{
14 One can confirm that a necessary condition for the two inequalities to hold jointly —and, thus, for an interior ESS to exist-is that $A_{C 2}+A_{D 2}>A_{C 1}+A_{D 3}$.
} 


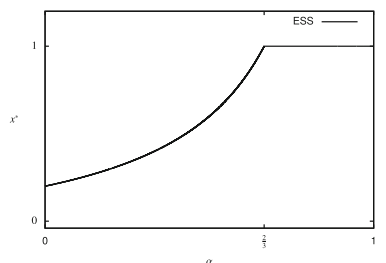

(a) $\mathrm{HD}$

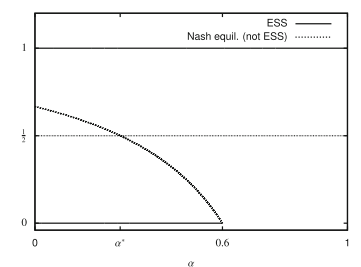

(b) $\mathrm{SH}$

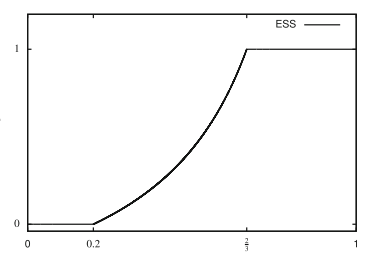

(c) PD (sub-additive)

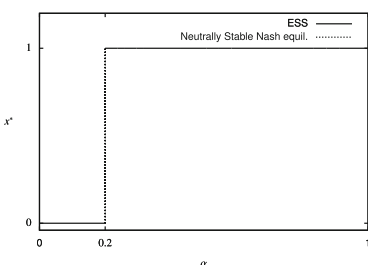

(d) PD (linear)

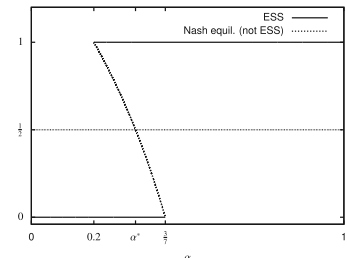

(e) PD (super-additive)

Fig. 1 Nash equilibria as a function of the index of assortativity for the games of Table 3

the population follows $C$ and another part of the population follows $D$ (polymorphic equilibrium). Finally, the case where $A_{D 2}+A_{C 2}=A_{C 1}+A_{D 3}$ (linear PD) is a transitional case and includes a continuum of neutrally stable Nash equilibria for a particular value of the assortativity parameter. These results can be seen in Fig. 1.

Risk Dominance In the case of normal form games with $A_{D 2}+A_{C 2}<A_{C 1}+A_{D 3}$, there is a value of the index of assortativity $\alpha^{*}=\left(\left(A_{D 2}-A_{C 2}\right)-\left(A_{C 1}-A_{D 3}\right)\right) /$ $\left(\left(A_{D 2}-A_{C 2}\right)+\left(A_{C 1}-A_{D 3}\right)\right)$ for which the basin of attraction of the ESS at $x=1$ is greater than that of the ESS at $x=0$ iff $\alpha \in\left(\alpha^{*}, 1\right]$. We can interpret that as follows. Assume that individuals in the population do not know whether each of the other players is going to play $C$ or $D$ and so, using the principle of insufficient reason, they ascribe equal probabilities (equal to 0.5 each) to each other player following $C$ and $D .{ }^{15}$ Then, if $\alpha \in\left(\alpha^{*}, 1\right]$ the expected payoff for a player following $C$ is higher than his expected payoff when he follows $D$ and so, given the aforementioned beliefs, it is a best response for all of them to follow $C$, leading to the population state being $x=1$. Conversely when $\alpha \in\left[0, \alpha^{*}\right)$.

So, in the terms described above, we can have a notion of risk dominance in the induced population game. Of course, as in both the $\mathrm{SH}$ and the super-additive PD it is true that $A_{D 2}+A_{D 3}>A_{C 1}+A_{C 2}$, when $\alpha=0$ (uniformly random matching) the risk dominant equilibrium is the one where the whole population follows $\mathrm{D}(x=0)$.

Efficiency When faced with a normal-form game payoff matrix, one might ask what the population state $x^{*}$ that maximizes average fitness (under uniformly random matching) is. ${ }^{16}$ One might then try to achieve efficiency by naïvely implementing $x^{*}$ as a Nash

\footnotetext{
15 See also Carlsson and Van Damme (1993).

16 Notice that for our $2 \times 2$ games this average fitness will coincide with the expected payoff in the normal form game when both players use that same strategy.
} 


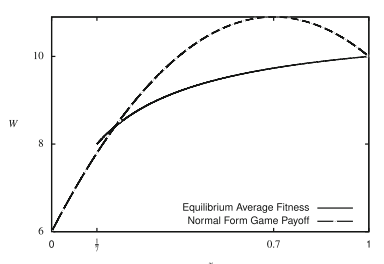

(a) HD

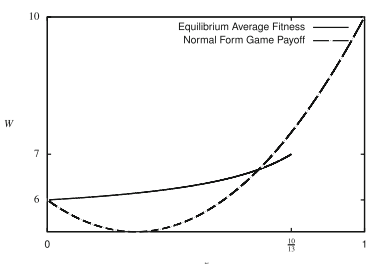

(b) $\mathrm{SH}$

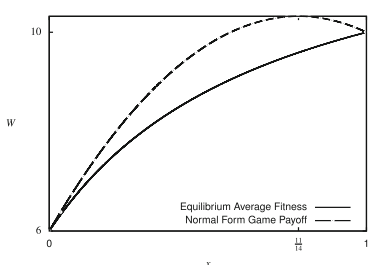

(c) PD (sub-additive)

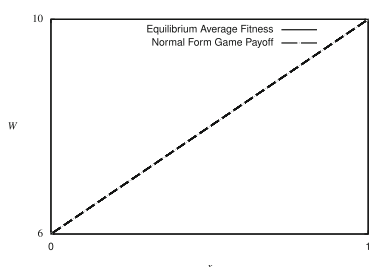

(d) PD (linear)

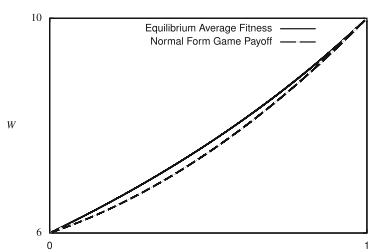

(e) PD (super-additive)

Fig. 2 Equilibrium average fitness and normal form game payoff for the games of Table 3

equilibrium through the application of an appropriate matching rule. The problem in the above is that if the rule that needs to be used is non-uniformly random, then the average fitness in equilibrium will (generically) differ from the one calculated in the beginning and may also no longer be optimal (maximum). We make such efficiency comparisons for our selected classes of games in what follows.

In order to conduct efficiency analysis, we use the methodology described in section A.3 of the Appendix. The comparison between Nash equilibrium average fitness in the induced game $F^{A, f}$ and expected payoff in the normal form game when both players use the same strategy for our class of games is shown in Fig. 2. Notice that in the HD and SH cases the equilibrium efficiency curve is not defined for some values of $x$ as these states cannot be attained as Nash equilibria of $F^{A, f}$ under any matching rule $f$.

In all our classes of games, the level of Nash equilibrium average fitness is strictly increasing with the proportion of $C$-individuals in the population and thus, Nash equilibrium efficiency is achieved when the Nash equilibrium is $x=1$ i.e. when the whole population follows $C$. Now, in the case where $A_{C 2}+A_{D 2} \leq 2 A_{C 1}$ (which is true for all SH, super-additive PD, and linear PD games), maximum Nash equilibrium average fitness coincides with the maximum expected payoff players using symmetric strategies can get in the normal form game (which is attained when both players play $C$ with certainty). In the case where $A_{C 2}+A_{D 2}>2 A_{C 1}$ (which can only hold for some $\mathrm{HD}$ and some sub-additive PD games), the normal form game maximum expected payoff (under symmetric strategy profiles) is obtained if both players play $C$ with probability $p_{C}^{*}=\frac{A_{C 2}+A_{D 2}-2 A_{D 3}}{2\left(A_{C 2}+A_{D 2}-A_{C 1}-A_{D 3}\right)} \cdot{ }^{17}$ However, when a matching rule that makes $x=p_{C}^{*}$ an equilibrium is implemented, equilibrium average fitness is reduced below

\footnotetext{
17 Note that PD games of this class are also referred to as Missing Hero Dilemmas in the literature (e.g. Nax and Rigos 2016; Diekmann and Przepiorka 2015).
} 
$A_{C 1}$. This is because the proportion of $C D$ pairs-which are efficient in the utilitarian sense-is reduced in favor of more $D D$ and $C C$ pairs which are not as efficient.

\section{Discussion}

Despite some similarity between the two settings, our results differ from those of Alger and Weibull (2013) due to the different nature of the strategy sets and, conseqently, of the assortativity considered. ${ }^{18}$ In particular, even in their "finite games" example, where they analyze $2 \times 2$ normal-form games, Alger and Weibull (2013) take the set of mixed strategies (an infinite, convex set) as the relevant strategy set. An evolutionarily stable strategy in their model is a mixed strategy $s \in \Delta_{m}$ such that if the whole population uses $s$, it cannot be invaded by a (uniform) population using any other mixed strategy $s^{\prime} \in \Delta_{m}$. The index of assortativity is then defined based on differences of probabilities of residents and invaders to encounter a resident i.e. between mixed strategies. In our model, assortativity is between pure strategies, that are the only ones available to the population. Assortative matching between mixed strategies makes payoffs to the two types nonlinear in the population state. Such nonlinearities cannot be captured in our pure-strategy case (as is shown in Table 4).

One could think that we could recover the results of Alger and Weibull (2013) if the resident population was considered to be using a pure strategy, say $C$. Even in this case our results differ though, since Alger and Weibull (2013) assume that the population withstands invasion from strategies arbitrarily close to the resident one whereas in our setting, the only possible invading strategy would be the "other" strategy $D$. Of course, since they only consider stability of homogeneous populations, any polymorphic equilibrium where both strategies are present in the population (see for example the HD game above) is excluded in Alger and Weibull (2013) as that would automatically render both strategies evolutionarily unstable.

\section{Conclusion}

This paper had two main purposes. Firstly, to extend the existing machinery of evolutionary game theory to include non-uniformly random matching under arbitrary matching rules and group sizes; and secondly, to discuss the relationship between matching and equilibrium efficiency. In Sect. 3.1 we showed that several results that hold for Nash equilibria and evolutionarily stable strategies under uniformly random matching extend to our setting (as one would expect from the literature on population games). As for efficiency, our main result (Proposition 3) showed that any evolutionary optimum will be a Nash equilibrium of the induced population game under some matching rule.

Often, matching is a geographical phenomenon: think of viruses, neighborhood imitation amongst humans, or trait-group/haystack-model-type of interactions (Cooper and Wallace 2004; Maynard Smith 1964; Wilson 1977). But when matching rules

18 We thank an anonymous referee for pointing out that different results are obtained in the two settings. 
correspond to institutions or conventions, not explaining how they come about misses half the story. A clear weakness of most existing models-including the results in this paper-is in this connection that the matching rules are taken as given. An obvious topic for future research would be to model the evolution of the matching rules (i.e., to endogenize them). An example of such an attempt is Nax and Rigos (2016) who endogenize the matching process via allowing individuals to vote for either more or less assortativity. Another direction could involve monitoring: If individuals gain an advantage by increasing their ability to monitor (by increasing their intelligence and memory), we can see how matching rules will over time evolve to be less and less random (typically more and more assortative). This then would be a true endogenous description of matching (institutions, conventions). The simplicity of the framework presented in this paper should definitely put such theories of evolving matching rules within reach.

Open Access This article is distributed under the terms of the Creative Commons Attribution 4.0 International License (http://creativecommons.org/licenses/by/4.0/), which permits unrestricted use, distribution, and reproduction in any medium, provided you give appropriate credit to the original author(s) and the source, provide a link to the Creative Commons license, and indicate if changes were made.

\section{Appendix}

\section{A Finding all Nash equilibria in $2 \times 2$ induced games}

In this section we provide a tool that makes it easy for one to find and visualize Nash equilibria and ESS of induced population games in the 2-player, 2-strategy case. Using our method, we can easily identify Nash equilibria of such games by looking for intersections between two lines: one that depends on the payoffs (the equilibrium curve) and one that depends on the matching rule in effect (the matching rule curve). An example is shown in Fig. 3; the Nash equilibrium is at the intersection of the two lines.

In what follows, we analyze games that have a payoff bimatrix of the general form presented in Table 5. For consistency with the analysis of Sect. 4, we will call

Fig. 3 Example of finding the Nash equilibrium of an induced game

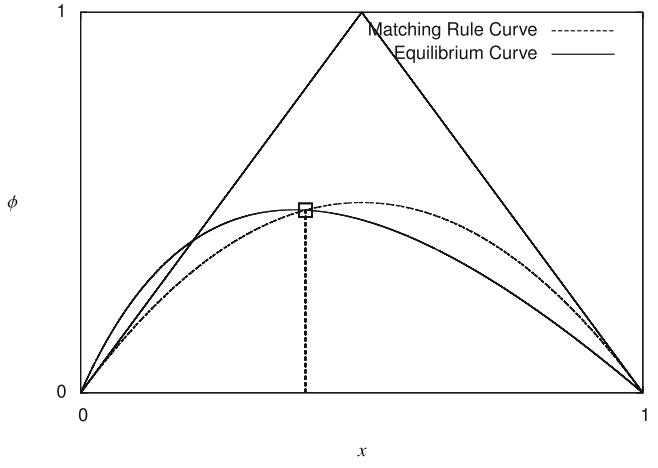


Table 5 The general form of a $2 \times 2$ game $\left(A_{C 1} \geq A_{D 3}\right)$

\begin{tabular}{lll}
\hline & $\mathrm{C}$ & $\mathrm{D}$ \\
\hline $\mathrm{C}$ & $A_{C 1}, A_{C 1}$ & $A_{C 2}, A_{D 2}$ \\
$\mathrm{D}$ & $A_{D 2}, A_{C 2}$ & $A_{D 3}, A_{D 3}$ \\
\hline
\end{tabular}

the two strategies $C$ and $D$ and we will use numbers to index the groups such that group-type 1 contains two $C$-type individuals, group-type 2 contains a $C$-type and a $D$-type individual and group-type 3 contains two $D$-type individuals. Without loss of generality, we will assume that $A_{C 1} \geq A_{D 3}$.

\section{A.1 The matching rule curve}

A matching rule for the $2 \times 2$ case, will be of the form $f(x)=\left(f_{1}\left(x_{C}, x_{D}\right), f_{2}\left(x_{C}, x_{D}\right)\right.$, $f_{3}\left(x_{C}, x_{D}\right)$ ). Now notice that as $f$ needs to satisfy (1) (two linearly independent equations in our example of 2 strategies), it can be easily described by only defining one of the three coordinates $f_{g}$. We pick the coordinate $f_{2}$-that expresses the extent to which the two strategies get mixed with one another - to describe the matching rule. Moreover, as there are only two strategies available, the population state can be summarized by the proportion of individuals using strategy $C$ as the remaining individuals are clearly using strategy $D$. We will use $x$ to denote this proportion and thus to express the population state. ${ }^{19}$ So any matching rule will be described by a function $\phi:[0,1] \rightarrow[0,1]$. Under the requirement $(1)$, the three coordinates of $f$ can be calculated to be:

$$
f_{1}(x)=x-\frac{1}{2} \phi(x) \quad f_{2}(x)=\phi(x) \quad f_{3}(x)=1-x-\frac{1}{2} \phi(x) .
$$

More than that, the conditions $f_{g}(x) \geq 0, g=1,2,3$ must be satisfied for all $x \in$ $[0,1]$. From these, we get that the values $\phi(x)$ can take are restricted by

$$
0 \leq \phi(x) \leq 2 x \text { for } x \in\left[0, \frac{1}{2}\right] \quad \text { and } \quad 0 \leq \phi(x) \leq 2(1-x) \quad \text { for } x \in\left(\frac{1}{2}, 1\right] \text {. }
$$

It is now possible to draw diagrams that show what matching rules look like. Examples of graphs of matching rules are given in Fig. 4. A matching rule is summarized by a line that begins at $(0,0)$, assumes values "within" the triangle bounded by $(15)$ and ends at $(1,0)$.

Under this formalization, the random matching rule will be given by

$$
\phi(x)=2 x(1-x)
$$

whereas the complete segregation rule is simply

$$
\phi(x)=0 .
$$

19 Obviously, $x_{C}=x$ and $x_{D}=1-x$. 
Fig. 4 Examples of matching rule curves

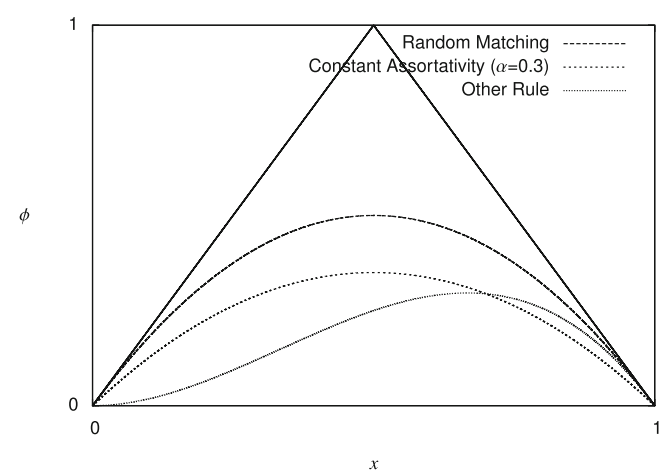

Another example would be the constant index of assortativity rule (Bergström 2003) which can be summarized by

$$
\phi(x)=2(1-\alpha) x(1-x)
$$

where $\alpha \in[0,1]$ is the index of assortativity.

\section{A.2 The equilibrium curve}

Under a matching rule $f$, a population state $x^{*} \in(0,1)$ is a Nash equilibrium of $F^{A, f}$ iff

$$
\begin{aligned}
F_{C}^{A, f}\left(x^{*}\right) & =F_{D}^{A, f}\left(x^{*}\right) \Leftrightarrow \\
{\left[\left(A_{D 2}-A_{D 3}\right) x^{*}+\left(A_{C 1}-A_{C 2}\right)\left(1-x^{*}\right)\right] \phi\left(x^{*}\right) } & =2\left(A_{C 1}-A_{D 3}\right) x^{*}\left(1-x^{*}\right) .
\end{aligned}
$$

Assuming that $\phi$ is differentiable at 0 and at 1 , we get the following conditions for boundary Nash equilibiria. For $x=0$ to be a Nash equilibrium, it must be the case that

$$
F_{C}^{A, f}(0) \leq F_{D}^{A, f}(0) \Rightarrow\left(A_{C 1}-A_{C 2}\right) \frac{\partial \phi}{\partial x}(0) \geq 2\left(A_{C 1}-A_{D 3}\right)
$$

and for $x=1$ to be a Nash equilibrium, it must be the case that

$$
F_{C}^{A, f}(1) \geq F_{D}^{A, f}(1) \Rightarrow\left(A_{D 3}-A_{D 2}\right) \frac{\partial \phi}{\partial x}(1) \leq 2\left(A_{C 1}-A_{D 3}\right) .
$$

Now, provided that there is actually some strategic interaction occurring between the two players, i.e. either $A_{D 2} \neq A_{D 3}$ or $A_{C 1} \neq A_{C 2}$ (or both), then from condition (16) we get two cases:

- If $A_{C 1} \neq A_{D 3}$, then $x \in(0,1)$ will be a Nash equilibrium iff $\phi(x)=E(x)$ where $E:[0,1] \rightarrow \mathbb{R}$ is defined by

$$
E(x)=\frac{2\left(A_{C 1}-A_{D 3}\right) x(1-x)}{\left(A_{D 2}-A_{D 3}\right) x+\left(A_{C 1}-A_{C 2}\right)(1-x)} .
$$




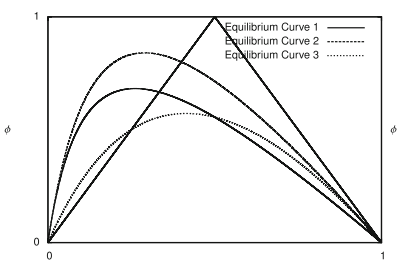

(a) HD

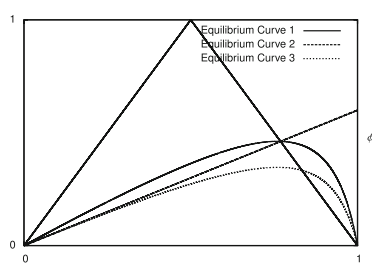

(b) $\mathrm{SH}$

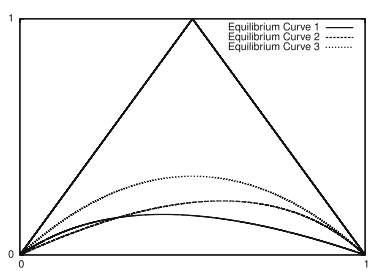

(c) $\mathrm{PD}$

Fig. 5 Equilibrium curves for our classes of games

We will call the graph of function $E$ the equilibrium curve of the game.

- In the case where $A_{C 1}=A_{D 3}$, then the condition for $x \in(0,1)$ to be a Nash equilibrium is

$$
\phi(x)=0 \quad \text { or } \quad x=\frac{A_{C 2}-A_{C 1}}{A_{D 2}-A_{D 3}+A_{C 2}-A_{C 1}} .
$$

Condition (20) says that any population state for which $C$-type and $D$-type individuals do not mix will be a Nash equilibrium (obviously, as no strategy gets an advantage over the other) and, more importantly, that the population state $\frac{A_{C 2}-A_{C 1}}{A_{D 2}-A_{D 3}+A_{C 2}-A_{C 1}}$ will be a Nash equilibrium for all matching rules (as long as this value is within the boundaries $(0,1))$.

Stability and the Equilibrium Curve If we assume that the matching rule is $C^{1}$ as is done for some of results stated in Sect. 3.1, then it can be easily verified that a population state $x$ will be an ESS iff

$$
\phi(x)=E(x) \text { and } \frac{\partial \phi}{\partial x}(x)>\frac{\partial E}{\partial x}(x) .
$$

As examples of the equilibrium curve analysis for our $2 \times 2$ games, we provide Fig. 5 that contains equilibrium curve examples for the classes of games analyzed in the main text.

Using the above analysis in conjunction with diagrams like the one in Fig. 4 can help us identify Nash equilibria and ESS very easily. All one has to do is to plot $\phi$ (which summarizes the matching rule) and the equilibrium curve $E$ (which summarizes the normal form game) on the same diagram. If the two lines meet at an interior population state $x$, then $x$ is a Nash equilibrium. If along with that the equilibrium curve is above the matching rule curve to the left of $x$ and below it to the right of $x$, then $x$ is an ESS as well. Finally, for the uniform population states $(x=0$ and $x=1)$, one can say that in order for one these to be a Nash equilibrium (ESS), then it has to be that the slope of the matching rule curve is greater than or equal to (greater than) the slope of the equilibrium curve at that population state. The Nash equilibrium-finding process for our classes of games is depicted in Fig. 6. 


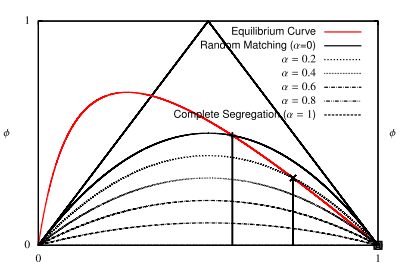

(a) HD

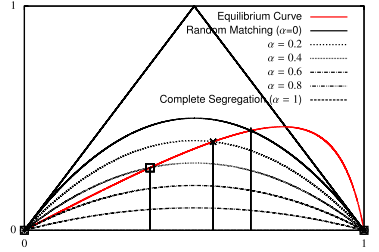

(b) $\mathrm{SH}$

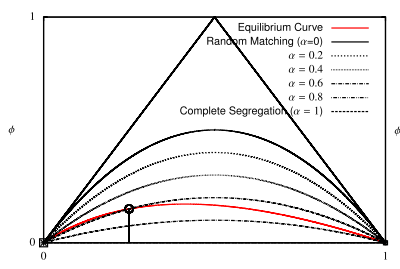

(c) PD (sub-additive)

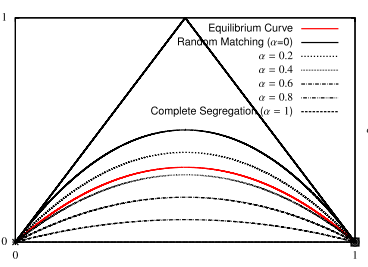

(d) PD (linear)

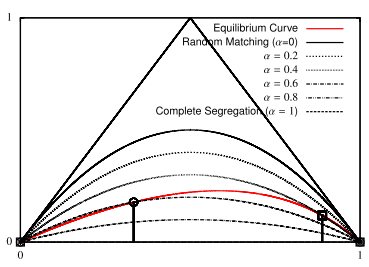

(e) PD (super-additive)

Fig. 6 The equilibrium-finding process for our classes of games

\section{A.3 Efficiency in $2 \times 2$ games}

In the case of $2 \times 2$ games, by using the formalization introduced above, we can make equilibrium efficiency considerations. We are interested in seeing how different Nash equilibria fare in terms of efficiency. For a $2 \times 2$ game, efficiency at state $x$ when the value of the matching rule at $x$ is $\phi$ is given by

$$
W(x, \phi)=A_{D 3}+\left(A_{C 1}-A_{D 3}\right) x+\frac{\left(A_{C 2}+A_{D 2}-A_{C 1}-A_{D 3}\right) \phi}{2}
$$

and as long as $A_{C 2}+A_{D 2} \neq A_{C 1}+A_{D 3}$, solving for $\phi$, we get

$$
\phi=\frac{2\left(W-A_{D 3}\right)}{A_{C 2}+A_{D 2}-A_{C 1}-A_{D 3}}-\frac{2\left(A_{C 1}-A_{D 3}\right) x}{A_{C 2}+A_{D 2}-A_{C 1}-A_{D 3}} .
$$

For any value of $W$, the above equation gives the set of points on the $(x, \phi)$ plane that yield an average payoff of $W$ for the population. We will call such lines isogrowth lines as all points on each of these lines lead to the same growth rate of the population (which is the same as the average payoff). Drawing such lines can help us visualize what is really happening in terms of efficiency under the various matching rules. More than that, by combining the isogrowth lines with the equilibrium curves of different games, we can see which matching rules lead to average fitness maximization. Isogrowth diagrams for the classes of games analyzed in the main text are shown in Fig. 7.

Finally, using the efficiency function (22) along with the equilibrium curve (19) we can calculate the Nash equilibrium efficiency in the induced game $F^{A, f}$ and then compare that to the expected payoff of a player in the normal form game when both players are using the same strategy. Such comparisons are carried out in Sect. 4 of the main text. 


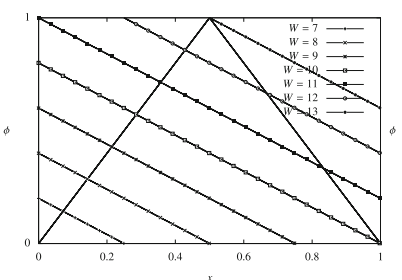

(a) $\mathrm{HD}$

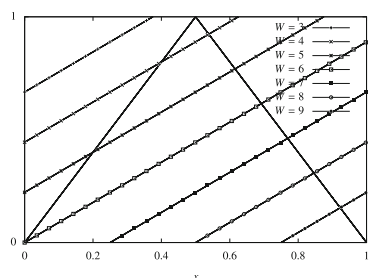

(b) $\mathrm{SH}$

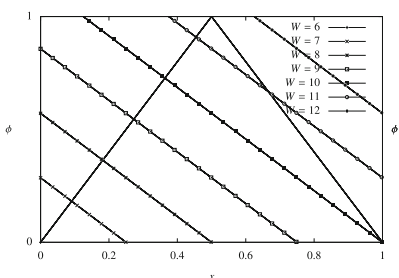

(c) PD (sub-additive)

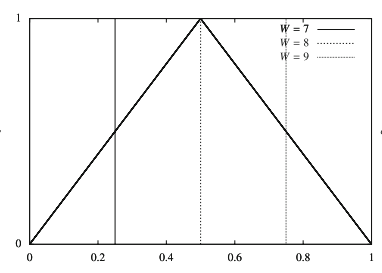

(d) PD (linear)

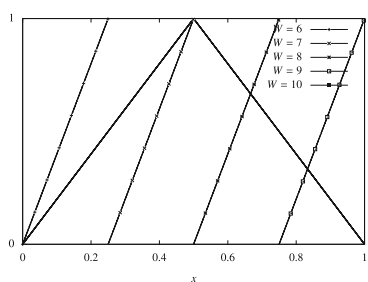

(e) PD (super-additive)

Fig. 7 Isogrowth diagrams for the games of Table 3

\section{B Omitted proofs}

\section{B.1 Proof of Proposition 2}

Let us denote by $y^{p} \in \Delta_{m}$ the (mixed) strategy used by player $p$ in the normal-form game $A$ and by $x^{-p} \in \Delta_{m}$ the strategy used in the normal-form game $A$ by all player $p$ 's opponents. Let also $U_{p}\left(y^{p} \mid x^{-p}\right)$ be the expected payoff of player $p$ in the normalform game when he/she is using strategy $y^{p}$ and all of his/her opponents use strategy $x^{-p} \in \Delta_{m}$. Since $A$ is symmetric, we have that $U_{p}(y \mid x)=P_{q}(y \mid x)$ for all players $p, q$. So we can write $U(y \mid x)$ to express the expected payoff in the normal-form game of any player using strategy $y$ when all his opponents use strategy $x$.

A symmetric Nash equilibrium of game $A$ is a strategy $x^{*} \in \Delta_{m}$ such that

$$
U\left(x^{*} \mid x^{*}\right) \geq U\left(y \mid x^{*}\right) \text { for all } y \in \Delta_{m}
$$

On the other hand, a strategy $x^{*}$ will be a Nash equilibrium in the induced game $F^{A, f}$ iff

$$
\Pi\left(x^{*}, x^{*}\right) \geq \Pi\left(y, x^{*}\right) \text { for all } y \in \Delta_{m} .
$$

In the above $\Pi\left(y, x^{*}\right)=y \cdot F^{A, f}\left(x^{*}\right)$ expresses the expected payoff to an individual using strategy $y$ while the rest of the population is using strategy $x^{*}$. In order to prove the proposition, all we need to show is that

$$
\Pi(y, x)=U(y \mid x) \text { for all } y \in \Delta_{m}
$$


under the uniformly random matching rule. If we let $e_{i}$ be the probability vector that corresponds to pure strategy $i$, then (24) boils down to

$$
F_{i}^{A, f}(x)=U\left(e_{i} \mid x\right) \text { for all } i \in M
$$

Calculating $F_{i}^{A, f}(x)$. Let us denote by $M_{-i}^{g}$ the set of strategies other than $i$ represented in group-type $g$, by $\Gamma_{i}^{1}$ the set of all group-types that contain exactly one individual of type $i$, and by $\Gamma_{i}$ the set of group-types that contain at least one individual of type $i$. Formally $M_{-i}^{g}=\left\{k \in M \backslash\{i\}: P_{i g}>0\right\}, \Gamma_{i}^{1}=\left\{g \in G: n_{i g}=1\right\}$, and $\Gamma_{i}=\left\{g \in G: P_{i g}>0\right\}$. Calculating $F_{i}^{A, f}(x)$ under the uniformly random matching rule yields

$$
F_{i}^{A, f}(x)=\sum_{g \in \Gamma_{i}} \frac{(n-1) ! x_{i}^{n_{i g}-1}}{\left(n_{i g}-1\right) !} \prod_{j \in M_{-i}^{g}} \frac{x_{j}^{n_{j g}}}{n_{j g} !} A_{i g} \quad \text { for } x \in \Delta_{m} \backslash \operatorname{bd}_{i}\left(\Delta_{m}\right)
$$

and

$$
F_{i}^{A, f}(x)=\sum_{g \in \Gamma_{i}^{1}}(n-1) ! \prod_{j \in M_{-i}^{g}} \frac{x_{j}^{n_{j g}}}{n_{j g} !} A_{i g} \quad \text { for } x \in \operatorname{bd}_{i}\left(\Delta_{m}\right) .
$$

Calculating $U\left(e_{i} \mid x\right)$. In general, in the normal-form game $A$, all players use mixed strategies i.e. a randomization over the set of pure strategies $M$. We will denote the pure strategy (action) a player $p$ ends up using after the randomization process has taken place-i.e. the realization of player $p$ 's mixed strategy-as $s^{p}$. The probability of a player ending up in a situation where his/her opponents follow (pure) strategies $s^{-p} \in M^{n-1}$ with $s^{-p}=\left(s^{1}, \ldots, s^{p-1}, s^{p+1}, \ldots, s^{n}\right)$ will be denoted by $\pi\left(s^{-p}\right)$. When all player $p$ 's opponents use the same strategy $x$, those probabilities can be calculated to be:

$$
\pi\left(s^{-p}\right)=\prod_{j=1}^{m}\left(x_{j}\right)^{v_{j}\left(s^{-p}\right)}
$$

where $v_{j}\left(s^{-p}\right) \in\{0,1, \ldots, n-1\}$ is the number of player $p$ 's opponents using strategy $j$ in realized profile $s^{-p}$.

Let us fix player $p$ 's strategy (realization) to be $s^{p}=i$. Since the game $A$ is symmetric, the payoff to player $p$ will not depend on the exact ordering in $s^{-p}$ but on the vector $v\left(s^{-p}\right)=\left(v_{1}\left(s^{-p}\right), \ldots, v_{m}\left(s^{-p}\right)\right)$. This means that different $s^{-p} \mathrm{~S}$ with the same $v\left(s^{-p}\right)$ will yield the same payoff for player $p$. Let us use $\kappa$ to index the different $v$. Abusing notation, we can calculate the probability of a specific $v^{\kappa}$ to occur to be

$$
\pi\left(\nu^{\kappa}\right)=\frac{(n-1) !}{\prod_{j=1}^{m} \nu_{j}^{\kappa} !} \prod_{j=1}^{m}\left(x_{j}\right)^{\nu_{j}^{\kappa}} .
$$

As player $p$ is using strategy $j$, if he ends up in a situation where his opponents' realizations are $\kappa$, it is as if he ends up in a group of type $g$ where $n_{j g}=v_{j}^{\kappa}$ for $j \neq i$ 
and $n_{i g}=v_{i}^{\kappa}+1$. Of course, for this group-type $P_{i g}>0$ will hold and we will write $g=i \triangleright \kappa$ and read: " $g$ is the group-type that we get if we add an individual who uses strategy $i$ to a set of opponents whose realizations are $\kappa$." Notice that the probabilities in (28) are independent of player $p$ 's choice of strategy. So, the probability of player $p$ ending up in situation $g$ conditional on him using strategy $i$ will be the same as the probability realization $\kappa$ occurring. Using the $g$-rather than the $\kappa$-indexing, we can rewrite (28) (abusing the notation once again) as

$$
\pi(g \mid i)=\pi(i \triangleright \kappa \mid i)=\pi\left(v^{\kappa}\right)=\frac{(n-1) ! x_{i}^{n_{i g}-1}}{\left(n_{i g}-1\right) !} \prod_{j \in M_{-i}^{g}} \frac{\left(x_{j}\right)^{n_{j g}}}{n_{j g} !} .
$$

Now, in each of these cases $g$, player $p$ gets a payoff of $A_{i g}$ and his expected payoff is

$$
U\left(e_{i} \mid x\right)=\sum_{g \in \Gamma_{i}} \pi(g \mid i) A_{i g}=\sum_{g \in \Gamma_{i}} \frac{(n-1) ! x_{i}^{n_{i g}-1}}{\left(n_{i g}-1\right) !} \prod_{j \in M_{-i}^{g}} \frac{\left(x_{j}\right)^{n_{j g}}}{n_{j g} !} A_{i g}
$$

In the special case where $x \in \operatorname{bd}_{i}\left(\Delta_{m}\right)$, player $p$ can be sure that he is the only one using strategy $i$ and thus, the only groups that get positive probability are the ones in $\Gamma_{i}^{1}$ which have $n_{i g}=1$. So his/her expected payoff is:

$$
U\left(e_{i} \mid x\right)=\sum_{g \in \Gamma_{i}^{1}} p(g \mid i) A_{i g}=\sum_{g \in \Gamma_{i}^{1}}(n-1) ! \prod_{j \in M_{-i}^{g}} \frac{\left(x_{j}\right)^{n_{j g}}}{n_{j g} !} A_{i g} .
$$

By comparing Eqs. (26)-(29) and Eqs. (27)-(30), we can see that

$$
F_{i}^{A, f}(x)=U\left(e_{i} \mid x\right)
$$

and as we showed that for an arbitrary $i$, it holds for all $i \in M$.

\section{B.2 Proof of Proposition 3}

Let $M^{g}=\left\{i \in M: g_{i}>0\right\}$ and let us define the following sets of group types:

$$
\begin{aligned}
\mathscr{E}\left(x^{*}\right) & =\left\{g \in G: x_{i}^{*}>0 \text { for all } i \in M^{g}\right\} \\
{[M] } & =\left\{g \in G: M^{g}=\{i\} \text { for some } i \in M\right\}
\end{aligned}
$$

$\mathscr{E}\left(x^{*}\right)$ consists of the group types that contain only individuals of types that are present in the population state $x^{*} . \mathscr{E}^{\prime}\left(x^{*}\right)$ will denote its complement i.e. group types that contain at least one individual of one of the types that are not present at $x^{*} .[M]$ consists of the groups types that contain only one type of individuals. We will denote the group type that contains only individuals of type $i$ by $[i]$. Now we can separate all group types in the following four categories: 
- $S P\left(x^{*}\right)=\mathscr{E}\left(x^{*}\right) \cap[M]$ is the set of all group types that contain a single type of individuals that are present at $x^{*}$.

- $S A\left(x^{*}\right)=\mathscr{E}^{\prime}\left(x^{*}\right) \cap[M]$ is the set of all group types that contain a single type of individuals that are absent at $x^{*}$.

- $M P\left(x^{*}\right)=\mathscr{E}\left(x^{*}\right) \backslash[M]$ is the set of all group types that contain more than one types of individuals that are present at $x^{*}$.

- $M A\left(x^{*}\right)=\mathscr{E}^{\prime}\left(x^{*}\right) \backslash[M]$ is the set of all group types that contain more than one types of individuals and at least one of them is absent at $x^{*}$.

Let us define for any $x \in \Delta_{m}$ the following:

$$
\mu=\arg \min _{i \in\left\{j \in M: x_{j}^{*}>0\right\}} \frac{x_{i}}{x_{i}^{*}}
$$

breaking ties for the smallest $i \in M$ that satisfies the condition. We construct $h$ as follows:

- For all $g \in M A\left(x^{*}\right)$ we define $h_{g}(x)=0$.

- For all $g \in S A\left(x^{*}\right)$ if $g=[i]$, we define $h_{g}(x)=x_{i}$.

- For all $g \in M P\left(x^{*}\right)$ we define $h_{g}(x)=\frac{x_{\mu}}{x_{\mu}^{*}} f_{g}^{*}\left(x^{*}\right)$.

- For all $g \in S P\left(x^{*}\right)$ if $g=[i]$, we define $h_{g}(x)=\frac{x_{\mu}}{x_{\mu}^{*}} f_{g}^{*}\left(x^{*}\right)+x_{i}-\frac{x_{\mu}}{x_{\mu}^{*}} x_{i}^{*}$.

It is easy to check that $h$ is a matching rule as it satisfies Definition 1. More than that, it is also easy to see that $h\left(x^{*}\right)=f\left(x^{*}\right)$ and so $\left(x^{*}, h\right)$ is an evolutionary optimum. All we have to do is to show that $x^{*}$ is a Nash equilibrium of $F^{A, f}$.

Now let us define $A^{*}=\max _{(x, f) \in \mathbb{E}} \bar{F}^{A, f}(x)$. As $\left(x^{*}, h\right)$ is an evolutionary optimum, it has to be that $x^{*}$ is a steady state of the replicator dynamics under $h$. So:

1. For all $i \in M$ with $x_{i}^{*}>0$ it has to be that $F^{A, h}\left(x^{*}\right)=A^{*}$ which is ensured by the fact that $h\left(x^{*}\right)=f\left(x^{*}\right)$ and

2. there is no restriction for $i \in M$ with $x_{i}^{*}=0$.

For $x^{*}$ to be a Nash equilibrium it must hold that:

$$
x^{*} \cdot F^{A, h}\left(x^{*}\right) \geq y \cdot F^{A, h}\left(x^{*}\right) \quad \text { for all } y \in \Delta_{m} .
$$

Notice that from point 1 above, if $x^{*} \in$ int $\left(\Delta_{m}\right)$, it is a Nash equilibrium as $y \cdot F_{h}^{A, f}\left(x^{*}\right)=A^{*}$ for all $y \in \Delta_{m}$ and the proposition holds.

If $x^{*} \in \operatorname{bd}\left(\Delta_{m}\right)$, then all we need to do is show that $F_{i}^{A, h}\left(x^{*}\right) \leq A^{*}$ for all $i$ with $x_{i}^{*}=0$. By definition

$$
F_{i}^{A, h}\left(x^{*}\right)=\sum_{g \in \Gamma_{i}} \frac{n_{i g}}{n} \partial_{i}^{+} h_{g}\left(x^{*}\right) A_{i g}=\partial_{i}^{+} h_{[i]}\left(x^{*}\right) A_{i[i]}+\sum_{g \in M A \cap \Gamma_{i}} \frac{n_{i g}}{n} \partial_{i}^{+} h_{g}\left(x^{*}\right) A_{i g}=A_{i[i]} .
$$

Finally, notice that under any matching rule the states $e_{i}=(0, \ldots, 0 \underbrace{, 1,}_{i \text {-th }} 0, \ldots, 0)$

are steady states and the payoff of all individuals on these states is simply: $\bar{F}^{A, h}\left(e_{i}\right)=$ $A_{i[i]}$. But as $\left(x^{*}, h\right)$ is an evolutionary optimum, we know that $A_{i[i]} \leq A^{*}$ for all $i \in M$. So, $F_{i}^{A, h}\left(x^{*}\right) \leq A^{*}$ for all $i \in M$. 


\section{References}

Aigner M (2007) A course in enumeration. Springer, Berlin

Alger I, Weibull JW (2010) Kinship, incentives, and evolution. Am Econ Rev 1725-1758

Alger I, Weibull JW (2012) A generalization of Hamilton's Rule-love others how much? J Theor Biol 299:42-54

Alger I, Weibull JW (2013) HomoMoralis_-preference evolution under incomplete information and assortativematching. Econometrica 81(6):2269-2302

Alger I, Weibull JW (2016) Evolution and KantianMorality. Games Econ Behav 98:56-67

Alós-Ferrer C, Ania AB (2005) The evolutionary stability of perfectly competitive behavior. Econ Theory 26:497-516

Bergström TC (2002) Evolution of social behavior: individual and group selection. J Econ Perspect 2(16):6788

Bergström TC (2003) The algebra of assortative encounters and the evolution of cooperation. Int Game Theory Rev 5(3):211-228

Bergström TC (2013) Measures of assortativity. Biol Theory 8(2):133-141

Carlsson H, Van Damme E (1993) GlobalGames and equilibrium selection. Econometrica 61(5):989-1018

Cooney D, Allen B, Veller C (2016) Assortment and the evolution of cooperation in a Moran process with exponential fitness. J Theor Biol 409:38-46

Cooper B, Wallace C (2004) Group selection and the evolution of altruism. Oxford Econ Papers 56(2):307

Diekmann A, Przepiorka W (2015) Punitive preferences, monetary incentives and tacit coordination in the punishment of defectors promote cooperation in humans. Sci Rep 5:17-52

Eshel I, Samuelson L, Shaked A (1998) Altruists, egoists, and hooligans in a local interaction model. Am Econ Rev 88(1):157-179

Fehr E, Gächter S (2000) Cooperation and punishment in public goods experiments. Am Econ Rev 90(4):980-994

Grafen A (1979) The Hawk-Dove game played between relatives. Anim Behav 27:905-907

Hamilton WD (1964) The genetical evolution of social behaviour. II. J Theor Biol 7(1):17-52

Hamilton WD (1970) Selfish and spiteful behaviour in an evolutionary model. Nature 228:1218-1220

Hines W, Maynard Smith J (1979) Games between relatives. J Theor Biol 79(1):19-30

Hofbauer J, Sigmund K (1998) Evolutionary games and population dynamics. Cambridge University Press, Cambridge

Kerr B, Godfrey-Smith P (2002) Individualist and multi-level perspectives on selection in structured populations. Biol Philos 17(4):477-517

Lefebvre M (2007) Applied stochastic processes. Springer, New York

Leininger W (2006) Fending off one means fending off all: evolutionary stability inquasi-submodular aggregative games. Econ Theor 29(3):713-719

Maynard Smith J (1964) Group selection and kin selection. Nature 201(4924):1145-1147

Maynard Smith J (1982) Evolution and the theory of games. Cambridge University Press, Cambridge

Maynard Smith J, Price GR (1973) The logic of animal conflict. Nature 246(5427):15-18

Nax HH, Murphy RO, Helbing D (2014) Stability and welfare of 'Merit-Based' group-matching mechanisms in voluntary contribution game. Available at SSRN 2404280

Nax HH, Rigos A (2016) Assortativity evolving from social dilemmas. J Theor Biol 395:194-203

Newton J (2017) The preferences of Homo Moralis are unstable under evolving assortativity. Int J Game Theory 46(2):583-589

Newton J (2018) Evolutionary game theory: a renaissance. Games 9(2):31

Nowak MA, May RM (1992) Evolutionary games and spatial chaos. Nature 359(6398):826-829

Ohtsuki H (2010) Evolutionary games in Wright's Island model: kin selection meets evolutionary game theory. Evolution 64(12):3344-3353

Pohley H-J, Thomas B (1983) Non-linear ESS-models and frequency dependent selection. Biosystems 16(2):87-100

Ritzberger K, Weibull JW (1995) Evolutionary selection in normal-formgames. Econometrica 63(6):13711399

Rousset F (2004) Genetic structure and selection in subdivided populations. Princeton University Press, Princeton

Samuelson L (2002) Evolution and game theory. J Econ Perspect 16:47-66 
Sandholm WH (2010) Population games and evolutionary dynamics (economic learning and social evolution). The MIT Press, Cambridge, Massachusetts

Schaffer ME (1988) Evolutionarily stable strategies for a finite population and a variable contest size. J Theor Biol 132:469-478

Skyrms B (2004) The Stag hunt and the evolution of social structure. Cambridge University Press, Cambridge Taylor PD, Jonker LB (1978) Evolutionary stable strategies and game dynamics. Math Biosci 40(1):145-156 Vega-Redondo F (1997) The Evolution ofWalrasian Behavior. Econometrica 65(2):375-384

Weibull JW (1995) Evolutionary game theory. The MIT Press, Cambridge Massachussets

Wilson DS, Dugatkin LA (1997) Group selection and assortative interactions. Am Nat 149(2):336

Wilson DS (1977) Structured demes and the evolution of group-advantageous traits. Am Nat 111(977):157185

Wright S (1921) Systems of mating. Genetics 6:111-178

Wright S (1922) Coefficients of inbreeding and relationship. Am Nat 56:330-338

Wu J (2016) Evolving assortativity and social conventions. Econ Bull 36(2):936-941

van Veelen M (2011) The replicator dynamics with n players and population structure. J Theor Biol 276(1):78-85

van Veelen M, García J, Rand DG, Nowak MA (2012) Direct reciprocity in structured populations. Proc Nat Acad Sci 109(25):9929-9934 